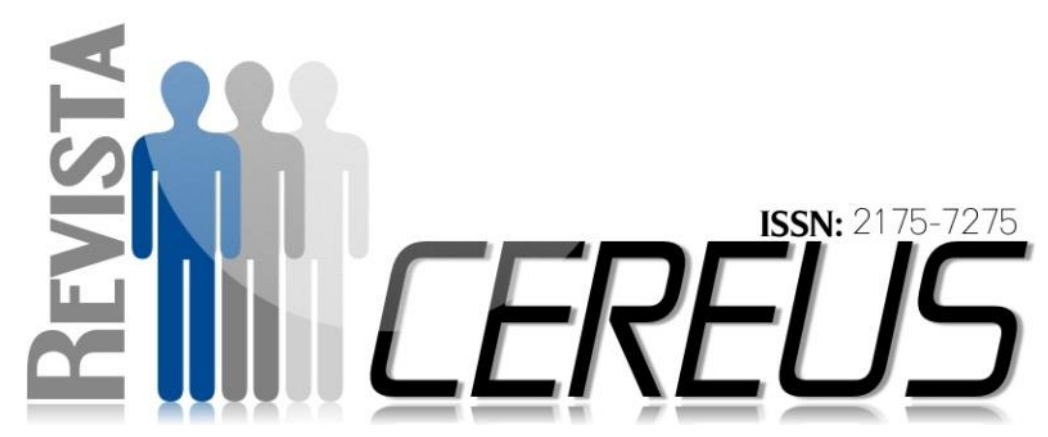

\title{
PERFIL DOS CASOS NOTIFICADOS DE HANSENÍASE NO MUNICÍPIO DE GURUPI/TOCANTINS POR MEIO DO SISTEMA DE INFORMAÇÃO DE AGRAVOS DE NOTIFICAÇÃO (SINAN)
}

\section{RESUMO}

\section{SIRTOLI, Daniela Bezerra ${ }^{1}$ GAMA, Pollyana Ferreira ${ }^{1}$ MARRONI, Márcia²}

A hanseníase é uma doença crônica e infecciosa, relevante para a saúde pública devido ao seu poder incapacitante. Em 2011, o Ministério da Saúde registrou no Brasil mais de 37 mil casos da doença 7\% em menores de 15 anos. Objetivou-se descrever o perfil dos casos de hanseníase distinguidos por sexo, notificados no Sistema de Informação de Agravos de Notificação (SINAN) no município de Gurupi-TO. Trata-se de um estudo epidemiológico descritivo, retrospectivo utilizando dados secundários do SINAN. Foram registrados 221 casos de hanseníase no período de 2013 a 2015, sendo 134 casos no sexo masculino e 87 casos no sexo feminino; Na faixa etária de 35-49 anos: 39 casos em homens e 23 casos em mulheres; Modo de entrada: os novos casos tiveram os

\footnotetext{
${ }^{1}$ Acadêmicas do curso de Enfermagem do Centro Universitário UnirG. Gurupi-TO. Emails: danielabsirtoli@gmail.com pollyanaferreiragama@hotmail.com 2 Professora Titular do Centro Universitário UnirG. Doutora em Saúde do Adulto pela Escola de Enfermagem da USP.
} 
maiores números notificados, sendo 111 casos em homens e 74 casos em mulheres; Modo de detecção por meio do encaminhamento: 65 casos em homens e 46 casos em mulheres; Lesões cutâneas: lesão única 48 casos em homens e 49 casos em mulheres; Avaliação diagnóstica: ignorados 102 casos em homens e 74 casos em mulheres; Classe Operacional: multibacilar 99 casos de homens e 45 casos de mulheres; Esquema Terapêutico Atual: PQT/MB/12 doses 91 casos de homens e 42 casos em mulheres; Tipo de Saída: Cura com 74 casos de homens e 63 casos de mulheres. Portanto verificou-se que a maioria de casos notificados de hanseníase foi no sexo masculino. Deve-se realizar um direcionamento maior das políticas públicas de saúde para a atenção a saúde do homem.

Palavras-chave: Hanseníase. Epidemiologia. Sexo.

\section{PERFIL DE CASOS NOTIFICADOS DE LA LEPRA EN LA CIUDAD GURUPI/TOCANTINS A TRAVÉS DEL SISTEMA DE INFORMACIÓN AGRAVACIONES NOTIFICACIÓN} (SINAN)

\section{ABSTRACT}

La lepra es una enfermedad crónica e infecciosa, relevante para la salud pública debido a su poder incapacitante. En 2011, el Ministerio de Salud registró en Brasil más de 37 mil casos de la enfermedad el 7\% en menores de 15 años. Se objetivó describir el perfil de los casos de hanseniasis distinguidos por sexo, notificados en el Sistema de Información de Agravios de Notificación (SINAN) en el municipio de Gurupi-TO. Se trata de un estudio epidemiológico descriptivo, retrospectivo utilizando datos secundarios del SINAN. Se registraron 221 casos de lepra en el período de 2013 a 2015, siendo 134 casos en el sexo 
masculino y 87 casos en el sexo femenino; En el grupo de edad de 35-49 años: 39 casos en hombres y 23 casos en mujeres; Modo de entrada: los nuevos casos tuvieron los mayores números notificados, siendo 111 casos en hombres y 74 casos en mujeres; Modo de detección a través del encaminamiento: 65 casos en hombres y 46 casos en mujeres; Lesiones cutáneas: lesión única 48 casos en hombres y 49 casos en mujeres; Evaluación diagnóstica: ignorados 102 casos en hombres y 74 casos en mujeres; Operación: multibacilar 99 casos de hombres y 45 casos de mujeres; En la mayoría de los casos, los casos de hombres y 42 casos en mujeres; Tipo de Salida: Cura con 74 casos de hombres y 63 casos de mujeres. Se observa que la mayoría de los casos notificados de lepra fue en el sexo masculino. Se debe realizar un direccionamiento mayor de las políticas públicas de salud para la atención a la salud del hombre.

Key Words: Lepra. Epidemiología. Género. 


\section{INTRODUÇÃO}

A hanseníase pode ocorrer em qualquer idade, raça ou gênero. A hanseníase, outrora conhecida como lepra e mal de Hansen, é uma doença crônica e infecciosa, relevante para a saúde pública devido ao seu grande poder incapacitante. A doença afeta, especialmente a pele e os nervos periféricos, mas pode expressar como uma doença sistêmica prejudicando as articulações, olhos, testículos, gânglios e outros órgãos. A elevada capacidade da hanseníase de impossibilitar seus portadores está diretamente associada ao potencial de penetração do Mycobacterium leprae na célula nervosa e seu poder imunogênico (BRASIL, 2008).

A hanseníase é causada por um bacilo resistente chamado de Mycobacterium leprae, é uma micobactéria intracelular obrigatória, sendo a única da espécie que causa infecção em nervos periféricos, em especial nas células de Schwann. Esse bacilo tem alta infectividade e baixa patogenicidade que dependem de características intrínsecas do bacilo, do hospedeiro e do nível de endemia do meio. A transmissão da hanseníase se da principalmente pela via aérea superior onde os bacilos são eliminados por pacientes multibacilares, sendo também a via de entrada do M. leprae no corpo. Após contato com esse bacilo o período de incubação pode durar em média de 2 a 7 anos (BRASIL, 2010).

Os principais sinais e sintomas são as manchas esbranquiçadas (hipocrômicas), acastanhadas ou avermelhadas, com alterações de sensibilidade ao calor, dor e tato; formigamentos, choques e câimbras que evoluem para dormência, pápulas, infiltrações, tubérculos, nódulos normalmente sem sintomas; diminuição ou queda de pêlos, localizada ou difusa, especialmente sobrancelhas; diminuição falta ou ausência de sudorese no local. As lesões normalmente iniciam com hiperestesia evoluem para ausência de sensibilidade e, desse momento em diante não coçam e o paciente refere dormência em qualquer parte do corpo (BRASIL, 2015).

De acordo com Brasil (2010) o coeficiente de detecção de casos novos de hanseníase no Brasil oscilou de 29,37/100.000 habitantes, em 2003, para 20,52/100.000 habitantes, em 
2008. A região Norte apresentou o maior número de casos, em seguida a região Centro-oeste, logo depois o Nordeste.

Os países com maiores incidência são os que estão em desenvolvimento ou tem condições precárias de higiene e superpopulação (Sociedade Brasileira de Dermatologia 2016). Em 2011, o Ministério da Saúde registrou no Brasil mais de 37 mil casos da doença, sendo $7 \%$ em menores de 15 anos.

No começo dos anos 1980, aproximadamente 11 a 15 milhões de pessoas no mundo apresentavam hanseníase, em comparação com o ano de 2010 estima-se que 212.00 pessoas tiveram a doença. Os casos

\section{METODOLOGIA}

$\begin{array}{ccr}0 & \text { presente } & \text { estudo } \\ \text { epidemiológico } & \text { descritivo }\end{array}$
retrospectivo, utilizou dados secundários obtidos no SINAN específico para casos de hanseníase na população do Município de GurupiTO, no período de 2013 a 2015. A base de dados foi adquirida no Departamento de Informática do SUS (DATASUS), constituído por todos os casos de hanseníase notificados e novos por ano reduziram de mais de 500.000 em 2003 para menos de 250.000 em 2010. A Organização Mundial de Saúde tem o objetivo de alcançar uma taxa de prevalência menor que 1 caso para cada 10.000 pessoas. Na América Latina, o Brasil tem a maior incidência principalmente na Amazônia ocidental (BOLOGNIA, 2015).

Em vista disso, estabelecemos como objetivo dessa pesquisa descrever o perfil dos casos de hanseníase por sexo, notificados no Sistema de Informação de Agravos de Notificação (SINAN) no município de Gurupi, Estado do Tocantins, Brasil, no período de 2013 a 2015.

confirmados em residentes do município de Gurupi- TO, através da Ficha Individual de Notificação/Investigação de Hanseníase, instrumento que é responsável por alimentar o SINAN.

O município de Gurupi faz parte do estado do Tocantins localizado na região Norte do país. Possui uma área de 1.836,091 km² com 83.707 habitantes em 2015. Atualmente é o 
terceiro maior município do Estado (Instituto Brasileiro de Geografia e Estatística, 2016).

O processamento e a análise dos dados foram feitos a partir dos

\section{RESULTADOS}

$\mathrm{Na}$ Tabela 1 verifica-se que foram registrados 221 casos de hanseníase no período de 2013 a 2015 no SINAN. Sendo 134 casos no sexo masculino e 87 casos no sexo feminino.

A Tabela 2 observa-se a distribuição de casos de hanseníase softwares TabNet (DATASUS) e Excel (Microsoft). Em seguida organizados em tabelas e gráficos para melhor representação. por faixa etária segundo o sexo, as faixas etárias com maiores frequências foram de 35-49 anos com 62 casos registrados, sendo 39 em homens e 23 em mulheres.

Tabela 1: Casos de hanseníase por ano de avaliação segundo sexo em Gurupi-TO no período de 2013 a 2015.

\begin{tabular}{c|c|c|c}
\hline PERÍODO & MASCULINO & FEMININO & TOTAL \\
\hline 2013 & 33 & 32 & 65 \\
\hline 2014 & 57 & 30 & 87 \\
\hline 2015 & 44 & 25 & 221 \\
\hline TOTAL & 134 & 87 & 69 \\
\hline
\end{tabular}

Fonte: Dados organizados pelas autoras a partir do SINAN - Sistema de Informações de Agravos de Notificação. MS/SVS/CGHDE - Coordenação Geral de Hanseníase e Doenças em Eliminação

Tabela 2: Casos de hanseníase por faixa etária e sexo, no município de Gurupi-TO no período de 2013 a 2015.

\begin{tabular}{c|c|c|c}
\hline FAIXA ETÁRIA & MASCULINO & FEMININO & TOTAL \\
\hline $5-9$ & 4 & 3 & 7 \\
\hline $10-14$ & 6 & 4 & 12 \\
\hline $15-19$ & 8 & 19 & 50 \\
\hline $20-34$ & 31 & 23 & 62 \\
\hline $35-49$ & 39 & 23 & 55 \\
\hline $50-65$ & 32 & 8 & 19 \\
\hline $65-79$ & 11 & 2 & 5 \\
\hline 80 e + & 3 & 4 & \\
\hline
\end{tabular}

Fonte: Dados organizados pelas autoras a partir do SINAN - Sistema de Informações de Agravos de Notificação. MS/SVS/CGHDE - Coordenação Geral de Hanseníase e Doenças em Eliminação. 
Tabela 3: Casos de hanseníase distribuídos por modo de entrada e sexo no município de Gurupi/-TO no período de 2013 a 2015.

\begin{tabular}{c|c|c}
\hline MODO DE ENTRADA & MASCULINO & FEMININO \\
\hline Casos novos & 111 & 74 \\
\hline $\begin{array}{c}\text { Transferência do mesmo } \\
\text { município }\end{array}$ & 3 & - \\
\hline $\begin{array}{c}\text { Transferência de outro município } \\
\text { (mesma UF) }\end{array}$ & 4 & 1 \\
\hline Transferência de outro estado & 4 & 1 \\
\hline Recidiva & 3 & 6 \\
\hline Outros ingressos & 9 & 87 \\
\hline TOTAL & 134 & 5 \\
\hline
\end{tabular}

Fonte: Dados organizados pelas autoras a partir do SINAN - Sistema de Informações de Agravos de Notificação. MS/SVS/CGHDE - Coordenação Geral de Hanseníase e Doenças em Eliminação..

$\mathrm{Na}$ Tabela 3 verifica-se a distribuição do número de casos de hanseníase segundo o sexo e modo de entrada. É possível observar que novos casos, sendo 111 em homens e 74 em mulheres foram os mais frequentes para ambos os sexos. Outros ingressos representaram 9 casos em homens e 6 casos em mulheres.

Conforme exposto na Figura 1, o modo de detecção da hanseníase mais comum foi por meio do encaminhamento, sendo 65 casos encaminhados referentes aos homens e 46 casos de mulheres; A demanda espontânea, representando 41 casos de homens e 27 casos de mulheres; Ignorado/em branco, com 23 casos de homens e 13 casos de mulheres; exame coletividade nenhum caso registrado de homem e 1 caso de mulher; e exame contatos 5 casos de homens e nenhum caso registrado de mulher.
A Tabela 4 apresenta a distribuição do número de casos de hanseníase por lesões cutâneas e avaliação diagnóstica. A lesão única foi a mais frequente, sendo 48 casos em homens e 49 casos em mulheres; entre 2 a 5 lesões, 33 casos em homens e 25 casos em mulheres; Em branco ou ignorado 24 casos em homens e 3 casos em mulheres; Sobre a avaliação diagnóstica observa-se que o maior número de casos foram ignorados sendo 102 casos em homens e 74 casos em mulheres; Grau zero, 20 casos de homens e 4 casos de mulheres; Grau I, 4 casos em homens e 1 caso em mulher; Grau II, 8 casos em homens e 8 casos em mulheres.

$\mathrm{Na}$ Tabela 5 observa-se a distribuição do número de casos de hanseníase por classe operacional e esquema terapêutico atual, onde a classe operacional com maior número de casos foi multibacilar, sendo 99 
casos em homens e 45 casos em

mulheres. Paucibacilar obteve os valores de 35 casos em homens e 42 casos em mulheres. Já o esquema terapêutico atual, predominou-se 0 Poliquimioterapia Multibacilar 12 doses (PQT/MB/12 doses), com 91 casos de homens e 42 casos em mulheres; Poliquimioterapia Paucibacilar 6 doses (PQT/PB/6 doses), 33 casos de homens e 35 casos de mulheres; Poliquimioterapia Multibacilar 24 doses (PQT/MB/24 doses), 10 casos de homens e 10 casos de mulheres.
$\mathrm{Na}$ Figura 2 verifica-se a distribuição de casos de hanseníase por tipo de saída. Os maiores casos foi devido a cura, com 74 casos de homens e 63 casos de mulheres; Em tratamento, 38 casos de homens e 15 casos de mulheres; Transferência para o outro município, 6 casos de homens e 4 casos de mulheres; Óbito, 3 casos de homens e 2 casos de mulheres; Erro diagnóstico, 3 casos em homens e 1 casos em mulher.

Figura 1: Distribuição de casos de hanseníase por modo de detecção segundo sexo no município de Gurupi-TO no período de 2013 a 2015.

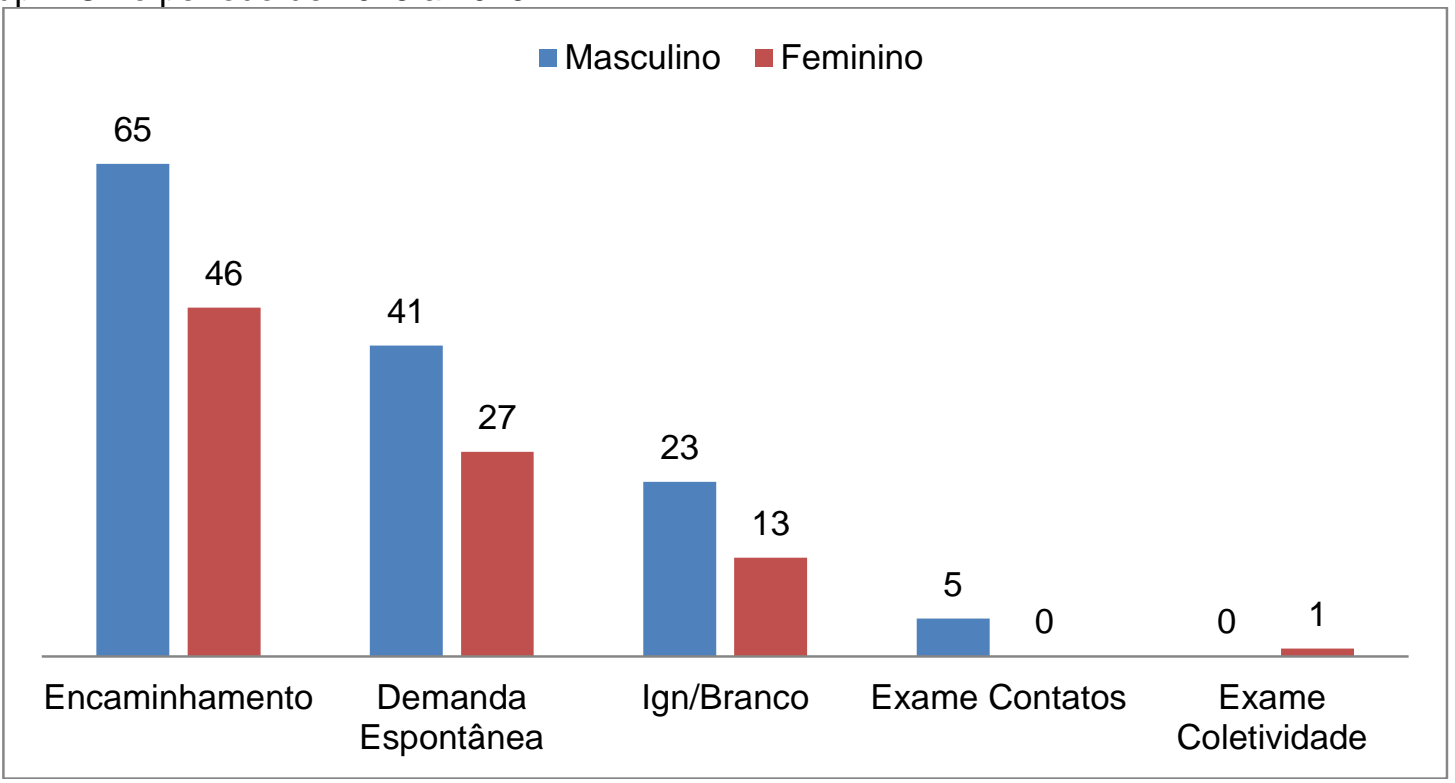

Fonte: Dados organizados pelas autoras a partir do SINAN - Sistema de Informações de Agravos de Notificação. MS/SVS/CGHDE - Coordenação Geral de Hanseníase e Doenças em Eliminação. 
Tabela 4: Distribuição do número de casos de hanseníase por lesões cutâneas e avaliação diagnóstica de acordo com o sexo no município de Gurupi/Tocantins no período de 2013 a 2015.

\begin{tabular}{ccc}
\hline LESÕES CUTÂNEAS & MASCULINO & FEMININO \\
\hline Nenhuma lesão & 11 & 3 \\
\hline Lesão única & 48 & 49 \\
\hline 2 a 5 lesões & 33 & 25 \\
\hline$>5$ lesões & 18 & 7 \\
\hline Branco ou Ignorado & 24 & 3 \\
\hline TOTAL & 134 & 87 \\
\hline AVALIAÇÃO DIAGNÓSTICA & & 74 \\
\hline Ignorado & 102 & 4 \\
\hline Grau zero & 20 & 1 \\
\hline Grau I & 4 & 8 \\
\hline Grau II & 8 & 87
\end{tabular}

Fonte: Dados organizados pelas autoras a partir do SINAN - Sistema de Informações de Agravos de Notificação. MS/SVS/CGHDE - Coordenação Geral de Hanseníase e Doenças em Eliminação

Tabela 5: Distribuição do número de casos de hanseníase por classe operacional e esquema terapêutico atual de acordo com o sexo no município de Gurupi/Tocantins no período de 2013 a 2015.

\begin{tabular}{ccc}
\hline CLASSE OPERACIONAL & MASCULINO & FEMININO \\
\hline Paucibacilar & 35 & 42 \\
\hline Multibacilar & 99 & 45 \\
\hline TOTAL & 134 & 87 \\
\hline $\begin{array}{c}\text { ESQUEMA TERAPÉUTICO } \\
\text { ATUAL }\end{array}$ & & \\
\hline PQT/PB/6 doses & 33 & 35 \\
\hline PQT/MB/12 doses & 91 & 42 \\
\hline PQT/MB/24 doses & 10 & 10 \\
\hline TOTAL & 134 & 87
\end{tabular}

Fonte: Dados organizados pelas autoras a partir do SINAN - Sistema de Informações de Agravos de Notificação. MS/SVS/CGHDE - Coordenação Geral de Hanseníase e Doenças em Eliminação

Figura 2: Distribuição de casos de hanseníase por tipo de saída segundo o sexo no município de Gurupi/Tocantins no período de 2013 a 2015.

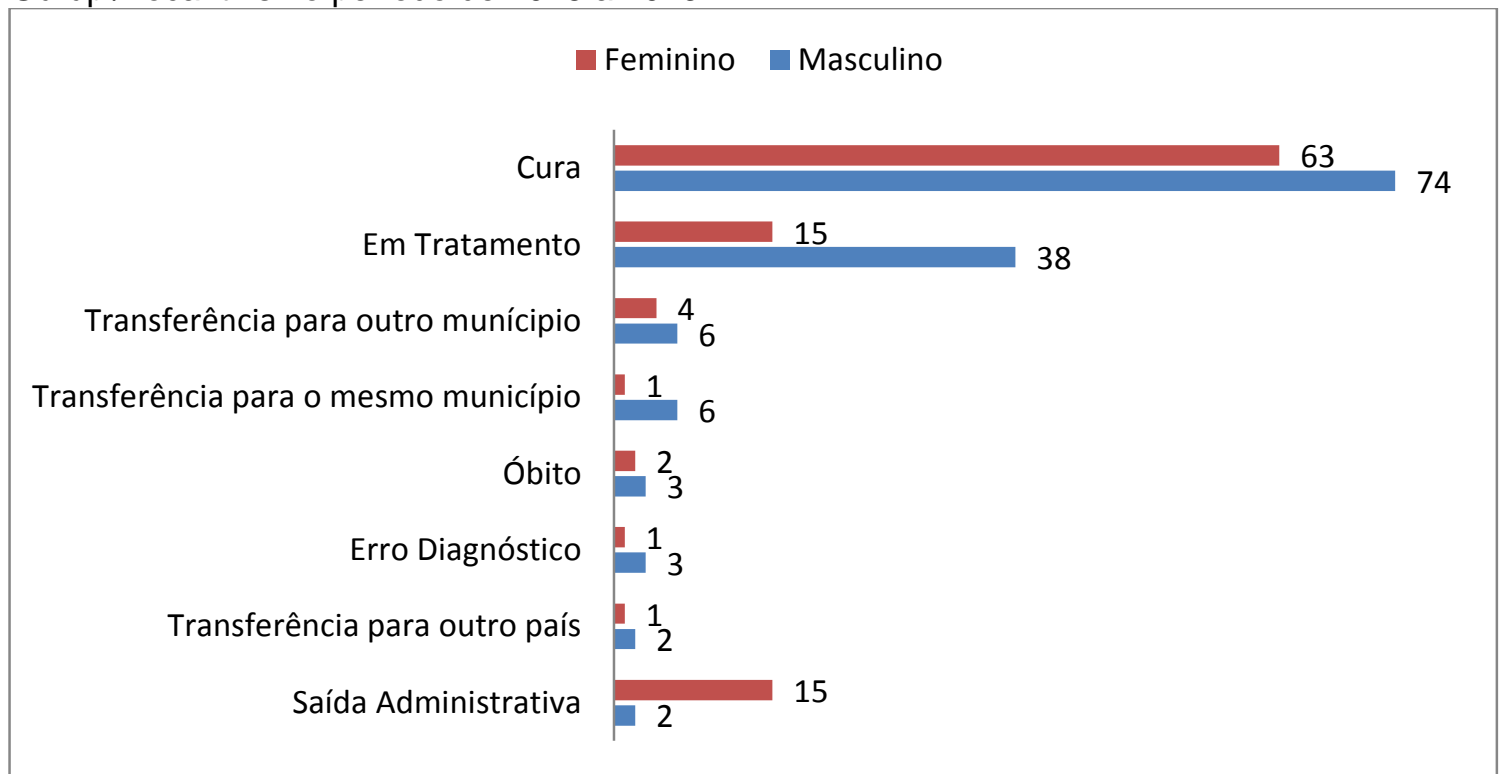

Fonte: Dados organizados pelas autoras a partir do SINAN - Sistema de Informações de Agravos de Notificação. MS/SVS/CGHDE - Coordenação Geral de Hanseníase e Doenças em Eliminação.

Rev. Cereus, v. 9, n. 3, p.184-196, set-dez./2017, UnirG, Gurupi, TO, Brasil. 


\section{DISCUSSÃO}

Apesar de poderem ser afetados igualmente pela hanseníase, os estudos mostram que os homens são afetados duas vezes mais que as mulheres (BOLOGNIA, 2015).

A hanseníase pode afetar todas as idades, no entanto pesquisas realizadas demostram um pico de incidência em duas faixas etárias: 10 a 15 e 30 a 60 anos de idade (BOLOGNIA, 2015).

De acordo com a Portaria SVS/MS no. 5 de 21 de fevereiro de 2006, todo caso confirmado de hanseníase deve ter notificação compulsória no Brasil. Todos os casos diagnosticados deverão ser notificados, utilizando a ficha de notificação e investigação do Sistema de Informação de Agravos de Notificação/Investigação (PORTALSAÚDE, 2016).

O diagnóstico dado para casos de hanseníase na Atenção Básica de Saúde é basicamente clínico através de exames dermatoneurológicos para detectar lesões ou áreas da pele com alteração de sensibilidade e comprometimento de nervos periféricos (BRASIL, 2008).

A Avaliação do Grau de Incapacidade precisa ser realizada obrigatoriamente em dois momentos no diagnóstico e na alta, como também a cada seis meses do tratamento. Esse procedimento é primordial para que se possam planejar ações de prevenção de incapacidades e para obter os indicadores epidemiológicos (BRASIL, 2008).

$\mathrm{Na}$ Classificação do Grau de Incapacidade temos o Grau zero, onde não se encontra nenhum problema com os olhos, mãos e pés devido à hanseníase. Grau I, tem a diminuição ou perda da sensibilidade nos olhos, mãos e/ou pés. Grau II, presenta de lagoftalmo e ectrópio; triquíase; opacidade corneana central; acuidade visual menor que 0,1 ou não conta dedos a 6m. Nas mãos existência de lesões tróficas e/ou lesões traumáticas; garras; reabsorção; mão caída. Nos pés presença de lesões tróficas e/ou traumáticas; garras; reabsorção; pé caído; contratura do tornozelo (BRASIL, 2008).

A Classificação operacional dos casos de Hanseníase é proposta para o tratamento com poliquimioterapia sendo baseada no número de lesões cutâneas que o indivíduo possa ter. Paucibacilar são os casos com até 5 
lesões cutâneas. Multibacilar são os casos com mais de 5 lesões cutâneas (BRASIL, 2010).

O tratamento da hanseníase é constituído por rifampicina, dapsona e clofazimina acondicionados em quatro tipos de cartelas de acordo com a classificação operacional de cada caso. A equipe da Unidade Básica de Saúde deve realizar 0 tratamento para hanseníase como parte de sua rotina, seguindo esquema terapêutico padronizado pelo Sistema Único de Saúde (SUS) (BRASIL, 2008).

A hanseníase por ser uma doença crônica, requer certo tempo de tratamento até que seja tenha o diagnóstico de cura. A cura do paciente só deve ser dada após uma avaliação médica. Após o individuo já ter tomado 6 doses das medicações em até 9 meses para os pacientes paucibacilar ou 12 doses tomadas em até 18 meses para os multibacilar. Não é raro que alguns pacientes evoluam para o óbito. Outro tipo de saída que pode haver é a transferência, onde o paciente sair da

\section{CONSIDERAÇÕES FINAIS}

Dado o objetivo do estudo em descrever o perfil dos casos de hanseníase por sexo, notificados esfera de responsabilidade da base geográfica que definiu a coorte. O paciente em tratamento é aquele que, por irregularidade, reiniciou o esquema terapêutico e está ainda em tratamento no momento da avaliação da coorte (BRASIL, 2008).

O homem no contexto da saúde sempre demostrou resistência a qualquer tipo de exame, consulta ou tratamento. Entre os homens existe uma falta de cultura preventiva que dê valor ao autocuidado, o medo de descobrir uma doença e a possibilidade de ter que realizar um tratamento, a vergonha em fazer procedimentos clínicos, como por exemplo, o toque retal e de se mostrar a um profissional da saúde homem ou mulher. São alguns elementos que tornam os homens mais susceptíveis a doenças, todos esses fatores devem ser observados pelos enfermeiros no planejamento e na execução das ações de promoção e prevenção da saúde (BRASIL, 2012).

SINAN no município de Gurupi-TO, no período de 2013 a 2015. Verificou-se que a maioria de casos notificados de 
hanseníase foi no gênero masculino. 0 homem no contexto da saúde sempre demostrou resistência a qualquer tipo de exame, consulta ou tratamento.

$\mathrm{O}$ estudo trouxe em um formato diferente a distribuição da hanseníase por sexo. Desse modo podemos analisar a hanseníase presente mais em homens do que em mulheres, na posse dessas informações, os profissionais da saúde podem criar ações voltadas para prevenção da saúde dos homens. Deve-se realizar um direcionamento maior das políticas públicas de saúde para a atenção a saúde do homem.
Há uma gama de conteúdo referente à hanseníase, mas pouca informação sobre população masculina que estar com hanseníase. Sugere-se que sejam realizados mais estudos sobre a adesão do homem aos tratamentos das doenças, em especial a hanseníase.

Dos 134 casos registrados de homens portadores de hanseníase e dos 87 casos em mulheres com hanseníase, 74 casos dos homens resultaram em cura e 63 casos das mulheres também obtiveram a cura.

\section{REFERÊNCIAS}

BOLOGNIA, Jean L. Dermatologia. [S.L.]: Disponível em: $<$ https://books.google.com.br/books?id=YwJACwAAQBAJ\&pg=PA1223\&dq=hansen $\%$ C3\%ADase\&hl=pt-

$\mathrm{BR} \& \mathrm{sa}=\mathrm{X} \&$ redir_esc $=\mathrm{y} \# \mathrm{v}=$ onepage\& $\mathrm{q}=$ hansen $\% \mathrm{C} 3 \% \mathrm{ADase} \& \mathrm{f}=$ false $>$. Acessado em 29 ago. 2016.

BRASIL. Ministério da Saúde. Secretaria de Atenção à Saúde. Departamento de Atenção Básica. Vigilância em Saúde: Dengue, Esquistossomose, Hanseníase, Malária, Tracoma e Tuberculose / Ministério da Saúde, Secretaria de Atenção a Saúde, Departamento de Atenção Básica. 2. ed. rev. Brasília: Ministério da Saúde, $2008 . \quad 195 \quad$ p. Disponível em: $<$ http://dab.saude.gov.br/docs/publicacoes/cadernos_ab/abcad21.pdf $>$. Acessado em 28 ago. 2016.

BRASIL. Ministério da Saúde. Secretaria de Atenção à Saúde. Departamento de Atenção Básica. Saúde do Homem no SUS. Ministério da Saúde, Secretaria de Atenção a Saúde, Departamento de Atenção Básica. 2. ed. rev. Brasília: Ministério da Saúde, 2012. 115 p. Disponível em: <http://portal.saude.sp.gov.br/resources/institutode-saude/homepage/bis/pdfs/bis_v14_1.pdf>. Acessado em 02 set. 2016. 
BRASIL. Ministério da Saúde. Secretaria de Vigilância em Saúde. Departamento de Vigilância Epidemiológica. Doenças infecciosas e parasitárias: guia de bolso/ Ministério da Saúde, Secretaria de Vigilância em Saúde, Departamento de Vigilância Epidemiológica. 8. ed. rev. Brasília: Ministério da Saúde, 2010. 448p. Disponível em: $<$ http://bvsms.saude.gov.br/bvs/publicacoes/doencas_infecciosas_parasitaria_guia_b olso.pdf>. Acessado em 28 ago. 2016.

BRASIL. Ministério da Saúde. Secretaria de Vigilância em Saúde. Departamento de Vigilância das Doenças Transmissíveis. Eliminar a hanseníase é possível: Um guia para os municípios. Coordenação-Geral de Hanseníase e Doenças em Eliminação. Brasília: Ministério da Saúde, 2015. 12p. Disponível em: < http://bvsms.saude.gov.br/bvs/publicacoes/eliminar_hanseniase_possivel_versao_pr eliminar.pdf>. Acessado em 28 ago. 2016.

INSTITUTO BRASILEIRO DE GEOGRAFIA E ESTATÍSCA. Gurupi, 2016. Disponível em: < https://cidades.ibge.gov.br/brasil/to/gurupi>. Acessado em 29 ago.. 2016.

PORTALSAÚDE. Hanseníase, 2016. Disponível em: $<$ http://portalsaude.saude.gov.br/index.php/o-ministerio/principal/leia-mais-oministerio/705-secretaria-svs/vigilancia-de-a-a-z/hanseniase/11294-descricao-dadoenca>. Acessado em 28 ago. 2016.

SOCIEDADE BRASILEIRA DE DERMATOLOGIA. Hanseníase, 2016. Disponível em: $<$ http://www.sbd.org.br/doencas/hanseniase/>. Acessado em 28 ago. 2016. 